\title{
Line tension of the pore edge in phospholipid/cholesterol bilayer from stretch molecular dynamics simulation
}

\author{
Taiki SHIGEMATSU*, Kenichiro KOSHIYAMA* and Shigeo WADA* \\ *Department of Mechanical Science and Bioengineering, Graduate School of Engineering Science, Osaka University, \\ 1-3 Machikaneyamacho, Toyonaka, Osaka 560-8531, Japan \\ E-mail: koshiyama@me.es.osaka-u.ac.jp
}

Received 31 July 2015

\begin{abstract}
The line tension of the pore in a phospholipid bilayer is important for pore-mediated molecular transport techniques. To understand the cholesterol effects on the line tension of the pore edge at the molecular level, we perform molecular dynamics simulations of phospholipid bilayers with a pore containing cholesterol in different concentrations $(0,20$, and $40 \mathrm{~mol} \%)$. The bilayer with a pore is prepared by using an equibiaxial stretching simulation. The stretched bilayer with a pore is subsequently compressed and the pore spontaneously closes when the applied areal strain of the bilayer is below a certain value. Using the pore closure areal strain and a free energy model of a stretched bilayer with a pore, the upper and lower limits of the line tensions for the bilayers containing cholesterol at 0,20 , and $40 \mathrm{~mol} \%$ are estimated to be 17.0-48.2, 54.5100 , and $170-261 \mathrm{pN}$, respectively. The increasing tendency of the line tension qualitatively agrees with that observed experimentally. The pores in the cholesterol-containing bilayers are lined with several cholesterol molecules, which might increase the bending rigidity of the pore edge, and result in the higher line tension of the cholesterol-containing bilayer. The considerable dependency of the line tension on the bilayer compositions might be useful to explain the large variations of the transduction efficiency observed with sonoporation treatment.
\end{abstract}

Key words : Cell membrane, Sonoporation, Electroporation, DPPC bilayer, Membrane rupture, Pore closure

\section{Introduction}

A phospholipid bilayer is a primary structure of the biological cell membrane, which separates the inside of the cell from the outside. Water-filled pores penetrating the bilayer are transiently formed by thermal fluctuations. Depending on the intensity of mechanical stress on the bilayer, the pore can spontaneously close or continue to grow. The pore in the bilayer acts as a path into the cell for extracellular molecules and enables passive molecular transport. The temporal permeabilization with the pore formation has been used as artificial molecular transport techniques, such as electroporation (Gehl, 2003) and sonoporation (Kinoshita and Hynynen, 2007; Kodama et al., 2009; Kudo et al., 2009). However, the excessive growth of the pore disturbs the cellular environments and induces rupture of the bilayer. For the safe and effective molecular transport, it is necessary to understand the details of pore dynamics in the bilayer under mechanical stresses.

Previously, to describe the pore dynamics, Litster proposed the free energy difference $\Delta F$ between bilayers with and without a pore with a radius $R$, as:

$$
\Delta F=2 \pi \gamma_{L} R-\pi \gamma_{S} R^{2},
$$

where $\gamma_{L}$ and $\gamma_{S}$ are the line tension (edge tension) and the surface tension, respectively (Litster, 1975). This model describes the free energy change with the change of the pore radius as the balance between the edge energy of the pore and the surface energy of the bilayer. From this model, the pore spontaneously closes when the pore radius is below $R_{C}$ $=\gamma_{L} / \gamma_{S}$, which is defined as the critical pore radius. However, when the pore exceeds $R_{C}$, the pore spontaneously grows, resulting in the rupture of the bilayer. Thus, the line tension $\gamma_{L}$, which governs $R_{C}$, is an important property of the bilayer and is useful for discussing the bilayer-edge-related phenomena, that is, rupture. 
The estimations of the line tension have been conducted by experimental (Karatekin et al., 2003; Portet and Dimova, 2010; Zhelev and Needham, 1993), theoretical (Akimov et al., 2014), and computational studies (Tolpekina et al., 2004; West et al., 2013; Wohlert et al., 2006). In these experiments, researchers have estimated the line tension from the pore closure dynamics in giant bilayer vesicles under stresses. These studies reported that the line tension depends on not only the lipid composition of the bilayer but also the purity of the lipids (Karatekin et al., 2003). In computational studies, molecular dynamics (MD) simulations of planar bilayer systems with a pore (Tolpekina et al., 2004) or bilayer ribbon system (West et al., 2013) have been performed. These studies clarified that the molecular details of the bilayer edge structure, and the line tensions estimated in MD simulations (10-50 pN) are in qualitative agreement with those obtained from experiments $(5-30 \mathrm{pN})$. In these MD simulation studies, pure phospholipid bilayers or mixture bilayers of phospholipids, which have different lengths of the hydrophobic tails (de Joannis et al., 2006; Wang et al., 2008), were used.

Actual cell membranes are intricately composed of various molecules. Among the various molecules in the cell membranes, cholesterol is especially known to be of importance. Cholesterol is abundant in mammalian cell membranes and the cholesterol concentration in phospholipid bilayers strongly depends on the type of the cell (Alberts et al., 1994). The cholesterol concentration is closely related with various properties of the bilayer; passive permeability (Demel, 1972), fluidity (Almeida et al., 1992), lateral distribution of membrane molecules (Pike, 2009), and mechanical stiffness (Needham and Nunn, 1990). In particular, experimental studies reported that the inclusion of cholesterol into the bilayer altered the line tension of the bilayer (Karatekin et al., 2003; Zhelev and Needham, 1993). Nevertheless, the details of the edge structure of cholesterol-containing bilayers at the molecular level and the relationship between the cholesterol around the pore edge and the line tension are still unclear. Furthermore, the edge structures at the molecular level are potentially related to the translocations of phospholipids between the inner and the outer leaflet of the bilayer (Gurtovenko and Vattulainen, 2007) and the permeations of ions and peptides trapped on the bilayer surface (Karal et al., 2015; Leontiadou et al., 2007). Thus, the molecular-level structure of the pore edge in the cholesterol-containing bilayer, which is a more realistic model for biological cell membranes than a pure bilayer, is a meaningful research target.

Herein, to understand the cholesterol effects on the line tension and the pore edge structure at the molecular level, we perform MD simulations of phospholipid/cholesterol bilayers with a pore under constant bilayer area conditions. The line tension of the cholesterol-containing bilayers is estimated from the free energy model of a bilayer with a pore proposed by Tolpekina and co-workers (2004). In their model, the line tension can be estimated from the critical condition that a pore spontaneously closes. Additionally, we present the potential relationships between the line tension of the bilayers and the efficiency of the pore-mediated molecular transport into cells.

\section{Methods}

\subsection{Estimation of line tension}

The line tension of the pore edge is estimated from MD simulations. For this purpose, we apply the method presented by Tolpekina et al. (2004) to our dipalmitoylphosphatidylcholine/cholesterol (DPPC/Chol) bilayer systems. In this method, to include the finite constant area in MD simulations, the free energy of the bilayer with a pore can be determined by:

$$
F=2 \pi \gamma_{L} R+\frac{K_{A}}{2 A_{0}}\left(\varepsilon_{A} A_{0}-\pi R^{2}\right)^{2},
$$

where $K_{A}$ is the area compressibility modulus (Tolpekina et al., 2004). $\varepsilon_{A}$ is the areal strain of the simulation box area calculated by $\left(A-A_{0}\right) / A_{0}$, where $A$ is the simulation box area and $A_{0}$ is the simulation box area without a pore at equilibrium. Here, we consider the equation $d F / d R=0$, whose roots are the stable or quasi-stable pore radii. When the discriminant of the cubic equation is negative, the equation has no positive root, which means that a pore will spontaneously close. The critical condition is given by:

$$
\varepsilon_{c}=3\left(\frac{\gamma_{L}}{2 K_{A}}\right)^{\frac{2}{3}}\left(\frac{\pi}{A_{0}}\right)^{\frac{1}{3}},
$$

where $\varepsilon_{c}$ is the critical areal strain where a pore spontaneously closes. We rewrite Eq. (3) as: 


$$
\gamma_{L}=2 K_{A}\left(\frac{\varepsilon_{c}^{3} A_{0}}{27 \pi}\right)^{\frac{1}{2}} .
$$

$K_{A}$ and $A_{0}$ can be obtained from MD simulation of the bilayers without a pore at equilibrium. We estimate the critical areal strain $\varepsilon_{c}$, where a pore is closed, by equilibrating MD simulations of stretched bilayers with a pore under various areal strain conditions. From these three parameters obtained in MD simulations and Eq. (4), we are able to estimate the line tension.

Bilayers with a pore are prepared through equibiaxial stretching simulations of intact bilayers. Stretch-induced pore formation was proposed to be more suitable for our purpose, the investigation of pore dynamics under mechanical stresses, than several other protocols used for making a pore employed in previous studies, such as self-aggregation (Leontiadou et al., 2004) and manual construction (Tolpekina et al., 2004; West et al., 2013). However, it is noted that the pore structures are very similar despite the different protocols used for the pore formation (Marrink et al., 2009). Thus, the selection of the protocols is expected to be a minor problem unless the pore formation process itself is discussed.

\subsection{Initial systems}

We use one pure DPPC bilayer system and two DPPC/Chol bilayer systems, as the force field and simulation protocols of DPPC bilayers have been well studied in MD simulations. These bilayers are composed of 200 DPPC and cholesterol molecules so that the cholesterol concentrations are 0,20 , and $40 \mathrm{~mol} \%$, respectively. These bilayers are set in the center of a square cylinder simulation box with periodic boundary conditions. The systems are solvated in at least 8284 water molecules. DPPC, cholesterol, and water molecules are represented by the united atom force fields for DPPC (Berger et al., 1997), for cholesterol (Höltje et al., 2001), and the simple point charge model for water (Berendsen et al., 1981). The initial configurations are taken from our previous study (Shigematsu et al., 2014). The systems were equilibrated at a temperature of $323 \mathrm{~K}$ and a pressure of $0.1 \mathrm{MPa}$ for at least $100 \mathrm{~ns}$ in our previous study (Shigematsu et al., 2014). The trajectories during the latter $50 \mathrm{~ns}$ are used for the analysis of the bilayers at equilibrium. Details of MD simulation parameters are summarized in the supplementary material of our previous study (Shigematsu et al., 2014) and the same parameters are used in our current investigation. All MD calculations are performed with the GROMACS molecular dynamics simulation codes (Berendsen et al., 1995; Hess and Kutzner, 2008). All snapshots of the bilayers are rendered by Visual Molecular Dynamics (VMD) (Humphrey et al., 1996).

\subsection{MD simulation outline}

To obtain the line tension $\gamma_{L}$ from Eq. (4), we estimate the critical areal strain $\varepsilon_{c}$, where a pore spontaneously closes, from the results of a series of MD simulations of bilayers with a pore. Herein, MD simulations are divided into three stages: stretching, compressing, and equilibrating stages (Fig. 1). The first stage is to induce the pore formation, the second is to modulate the applied areal strain of the system with a pore, and the third is to examine whether the pore closes under the constant areal strain conditions. Through these stages, MD simulations of the bilayers are performed with a thermostat (Bussi et al., 2007) and barostat (Berendsen et al., 1984) to maintain the temperatures of DPPC, cholesterol, and water molecules at $323 \mathrm{~K}$ and the pressure in the $z$-direction (normal to the bilayer) at $0.1 \mathrm{MPa}$. For the $x$ - and $y$-directions (bilayer plane), the box lengths are controlled to apply an arbitrary areal strain on the bilayer. The box lengths are elongated or shortened at a constant rate $c$, and, simultaneously, the atom positions are proportionally scaled to follow the changes of the box lengths (Koshiyama and Wada, 2011). $c$ is set to a positive value for stretching, negative for compressing, and zero for maintaining the bilayer area at a constant level.

In the stretching stage, the bilayer systems without a pore at equilibrium, taken from our previous study, are used for the initial configurations of this stage. Using these systems, we perform MD simulations with $c=1.0 \mathrm{~m} / \mathrm{s}$ until a pore is formed. It should be noted that, to induce the pore formation in conventional MD simulations, the bilayers have to be excessively stretched. This is believed to be because there is an energetic barrier between the bilayer with and without a pore (Abiror et al., 1979), which impedes the transition of the bilayer state from "without a pore" to "with a 
pore". In the MD simulations here, the pore formation occurs in the range of the areal strain from about 1 to 2 . The range is considerably larger than the critical areal strain $\varepsilon_{c}$, where a pore spontaneously closes $(<0.5)$.

In the compressing stage, the stretched bilayer with a pore just after the pore formation in the stretching stage is used for the initial configuration of this stage. To relax the excessive areal strain on the bilayer, we perform MD simulations with $c=-1.0 \mathrm{~m} / \mathrm{s}$ until the areal strain reaches 0 . As both the stretching and compressing stages occur at non-equilibrium, the bilayer system in the two stages has not yet reached a stable state nor a quasi-stable state.

In the equilibrating stage, we perform MD simulations with $c=0 \mathrm{~m} / \mathrm{s}$ for $100 \mathrm{~ns}$. The initial configuration of this stage is obtained from the trajectories in the compressing stage, where the box area is set to satisfy areal strains $\varepsilon_{A}=0.1$, $0.2,0.3,0.4,0.5$, and 0.6 . After the 100 -ns simulation in the equilibrating stage, we examine the existence of the pore from visual inspection and obtain the minimum areal strain where a pore remains in the bilayer and the maximum areal strain where a pore is no longer observed. The critical areal strain $\varepsilon_{c}$, where a pore spontaneously closes, is expected to be in the range between the two areal strains. Accordingly, we estimate the upper and lower limits of the line tension via $\mathrm{Eq}(4)$.

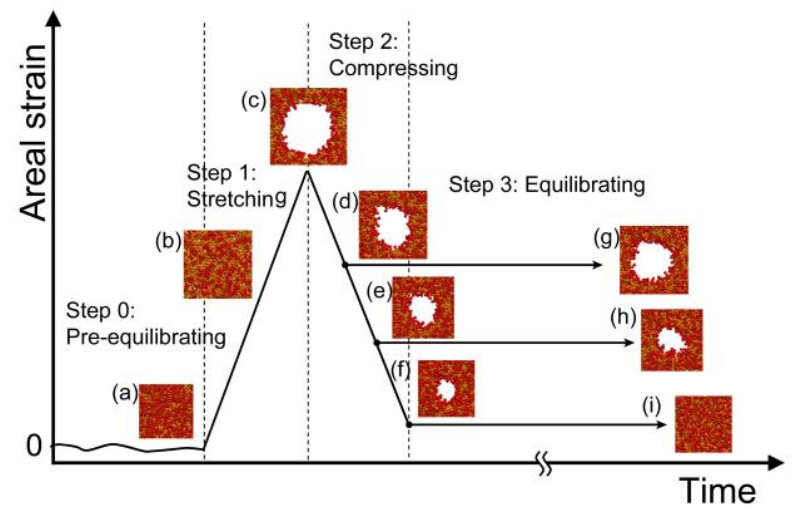

Fig. 1 Schematic of the outline of MD simulations of the pore formation and closure. Panels (a-i) are representative snapshots of the pure DPPC bilayer during stretching, compressing, and equilibrating stages. White regions in panels $(\mathrm{c}-\mathrm{h})$ are pores.

\subsection{Analysis}

In the bilayer without a pore, the bilayer area is considered to be equal to the simulation box area $A$. The area compressibility modulus of the bilayer $K_{A}$ is estimated from the fluctuations of $A$ at equilibrium by using:

$$
K_{A}=\frac{A_{0} k_{B} T}{\sigma_{A}^{2}},
$$

where $\sigma_{A}^{2}$ is the variance of $A, k_{B}$ is the Boltzmann constant, and $T$ is the temperature of the system. For the estimation of $K_{A}$, the trajectories in the pre-equilibrating stage obtained from our previous study (Shigematsu et al., 2014) are used.

As there are large fluctuations of the geometric structure of the pore in MD simulations, it is difficult to clearly distinguish the pore edge region of the bilayer. Herein, we define the region of the pore edge using the distance from the bilayer center along the $z$ axis (normal to the bilayer). The bilayer center is defined as the center of mass of the phosphorous atoms in DPPC molecules and the pore edge is defined as the region within which the distance is lower than $0.8 \mathrm{~nm}$ (region between the two dashed white lines in Fig. 4). A DPPC molecule, whose phosphorous atom is in the pore edge region, is considered to be in the pore edge region. As with the DPPC molecule, a cholesterol molecule is distinguished by the position of the hydrogen atom in the hydroxyl group.

\section{Results}

\subsection{Pore formation and closure}


In the stretching stage, owing to the stretching of the bilayer, the bilayer area increases and a pore is formed when the areal strain exceeds a critical value (Fig. $1(\mathrm{a}-\mathrm{c})$ ). The pore rapidly grows to a constant area, which depends on the applied areal strain (Fig. 1(c)). After the pore formation, the simulation proceeds to the compressing stage. In the compressing stage, owing to the compression, the area of the pore decreases; however, the pore is not closed only when the areal strain $\varepsilon_{A}$ reaches 0.0 (Fig. 1(c-f)). In the equilibrating stage, under lower areal strains, the area of the pore decreases gradually during the 100-ns equilibrium simulations, which resulted in the pore closure (Fig. 1(f) and (i)). However, under higher areal strains, the pore closure does not occur at least within the current simulation time (Fig. 1(d), (e), (g), and (h)). Figure 2 shows the snapshots of DPPC and DPPC/Chol bilayers taken from the end of the equilibrating stage. In the pure DPPC bilayer, the pore closure occurs with the areal strains $\varepsilon_{A} \leq 0.1$, and does not occur for $\varepsilon_{A} \geq 0.2$ (upper row in Fig. 2). In the DPPC bilayer with 20 and $40 \mathrm{~mol} \%$ cholesterol, the pore closure occurs for $\varepsilon_{A}$ $\leq 0.2$ and 0.3 , and does not for $\varepsilon_{A} \geq 0.3$ and 0.4 , respectively (middle and lower rows in Fig. 2). These indicate that $\varepsilon_{c} \mathrm{~s}$ for the bilayers are expected to be in the range of $0.1-0.2,0.2-0.3$, and $0.3-0.4$, respectively, and $\varepsilon_{c}$ increases with the increase of the cholesterol concentration (Fig. 3A).
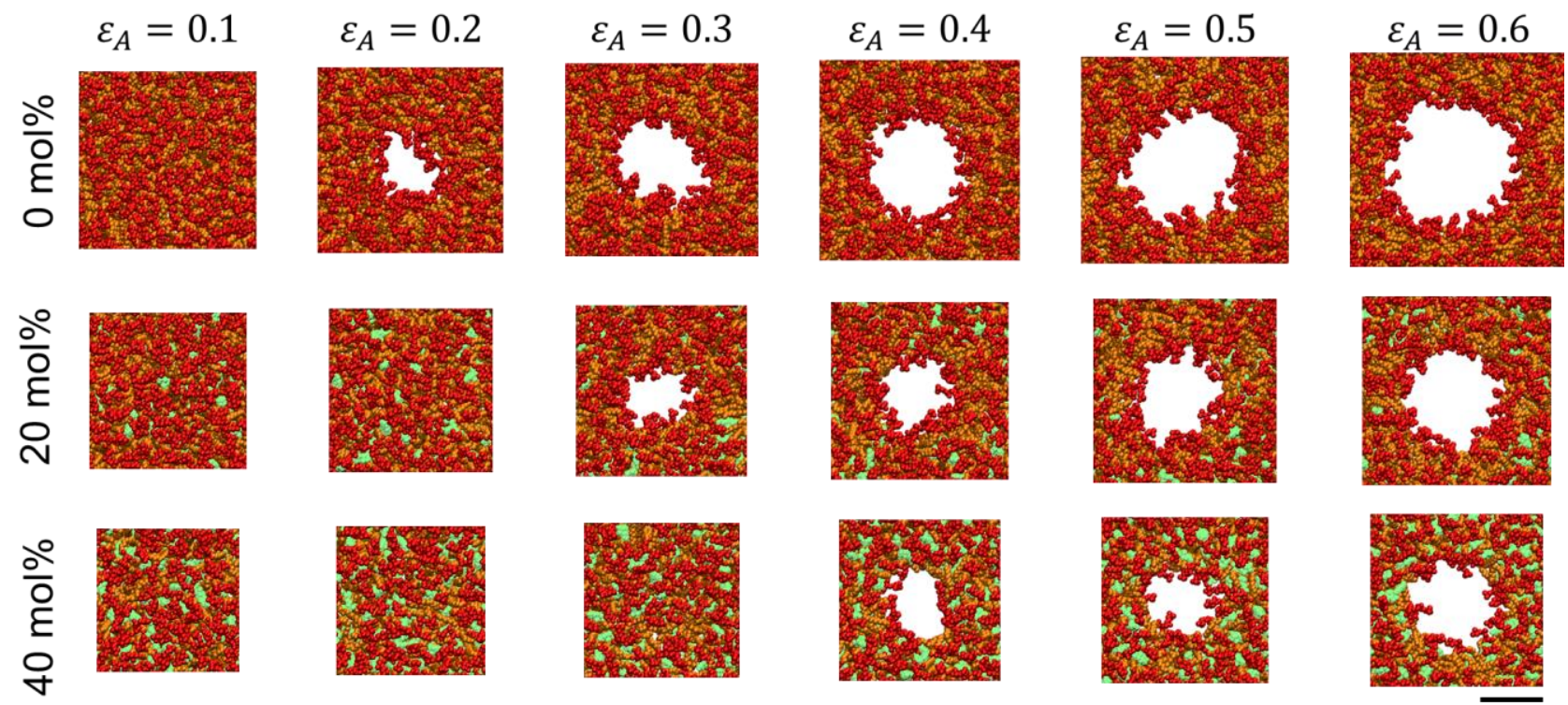

Fig. 2 Representative snapshots of the bilayers including cholesterol molecules at 0 (upper low), 20 (middle), and $40 \mathrm{~mol} \%$ (lower) after stretching, compressing, and equilibrating MD simulations. The column of the panels corresponds to the applied areal strain. DPPC head groups are shown in red, DPPC tails in orange, and cholesterol molecules in light green. Water molecules are not shown for clarity. White region at the center of the panels is a pore. The black bar on the bottom right corner corresponds to $3 \mathrm{~nm}$.
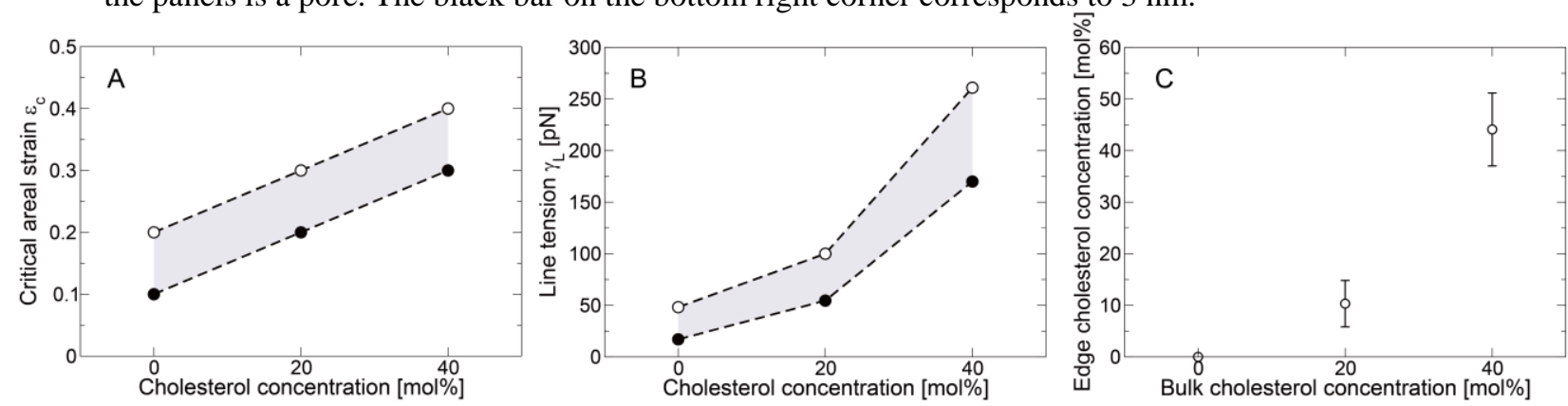

Fig. 3 Relationships between cholesterol concentration and critical areal strain (A), line tension (B), or cholesterol concentration in the pore edge region $(C)$. The dashed lines show the linearly interpolated lines between the points, and the region we expected to find the real critical areal strain and the line tension are shown in gray. Black bars in the panels A and B represent the estimated range within which are the critical areal strain or the line tension. Error bars in the panel $\mathrm{C}$ represent standard deviation. 


\subsection{Estimation of line tension}

From the trajectory of the bilayer at equilibrium taken from our previous study (Shigematsu et al., 2014), the averaged system box areas on the $x-y$ plain $A_{0}$ are $65.4,48.7$, and $39.2 \mathrm{~nm}^{2}$ for the bilayers containing cholesterol at 0 , 20 , and $40 \mathrm{~mol} \%$, respectively. The corresponding areas per molecule are $0.65,0.49$, and $0.39 \mathrm{~nm}^{2}$, respectively, which are in good agreement with those obtained from MD simulations reported by other groups (Chiu et al., 2002; Saito and Shinoda, 2011). Additionally, the variances of $A, \sigma_{A}^{2}$, are $0.95,0.54$, and $0.23 \mathrm{~nm}^{4}$ for the different cholesterol concentration bilayers, respectively. From $A_{0}, \sigma_{A}^{2}$, and Eq. (5), the area compressibility modulus $K_{A}$ can be calculated as 306,402 , and $760 \mathrm{mN} / \mathrm{m}$, respectively. $K_{A}$ increases with the increase of the cholesterol concentration, which is in qualitative agreement with that observed from experiments (Endress et al., 2002; Needham and Nunn, 1990). Using these parameters $A_{0}, K_{A}$, and $\varepsilon_{c}$ in Fig. 3A, the line tension $\gamma_{L}$ can be calculated by Eq. (4) and the relationships between the line tension and the cholesterol rate are shown in Fig. 3B. The upper and lower limits of the line tensions of the bilayers containing cholesterol at 0,20 , and $40 \mathrm{~mol} \%$ are estimated to be $17.0-48.2,54.5-100$, and $170-261 \mathrm{pN}$, respectively. The line tension of the pure DPPC bilayer is in agreement with those estimated by different methods in previous MD simulation studies (27.5-50 pN) (Leontiadou et al., 2004; West et al., 2013; Wohlert et al., 2006). The line tension increases with the increase of the cholesterol concentration, which qualitatively agrees with those for similar phospholipid bilayers reported from experiments (Karatekin et al., 2003; Zhelev and Needham, 1993).

\subsection{Pore edge structure}

Figure 4 shows the representative snapshots of the bilayers with stable pores, whose radii are similar. From a visual inspection, the pore edges are lined with a considerable number of DPPC molecules and several cholesterol molecules. The number of cholesterol molecules in the pore edge region increases with the increase of the cholesterol concentration in the whole system. Figure 3C shows the relationship between the cholesterol concentration in the pore edge region and that in the whole system. In the bilayer with $20 \mathrm{~mol} \%$ cholesterol, the edge cholesterol concentration is slightly lower than the cholesterol concentration of the whole system. However, in the bilayer with $40 \mathrm{~mol} \%$ cholesterol, the edge cholesterol concentration is slightly higher.
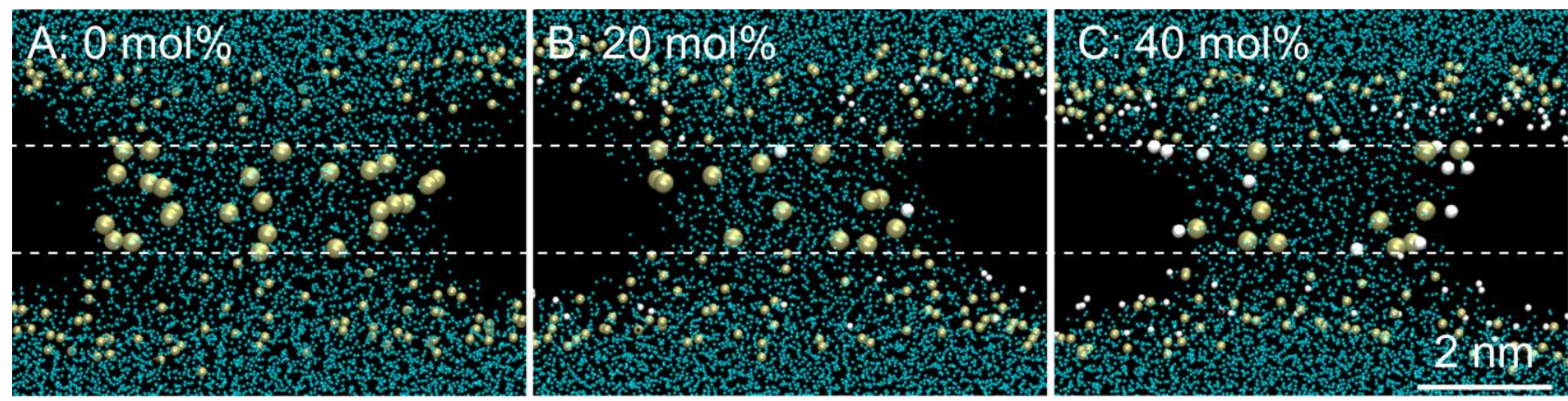

Fig. 4 Representative snapshots of the pore structures in the bilayer containing cholesterol at 0 (A), 20 (B), and 40 mol\% (C). Phosphorus atoms of DPPC molecules are shown as tan beads, hydrogen atoms of cholesterol molecules as white beads, and water molecules are blue. Dashed lines show the boundary between the pore edge region and the others. Phosphorous atoms and hydrogen atoms of cholesterol molecules in the pore edge region are enlarged for clarity.

\section{Discussion}

\subsection{Cholesterol effects on line tension}


From our simulation, the line tension increases with the increase of the cholesterol rate (Fig. 3B). We first compare our results with the experimental data. However, experimental data of the line tension in DPPC bilayers are non-existent to our knowledge, whereas DPPC bilayers are commonly used in MD simulation studies. Thus, experimental data for similar phospholipids are used to evaluate the cholesterol effects obtained in our MD simulation. In experimental studies, similar phospholipid molecules, for example, stearoyl-oleoyl phosphatidylcholine (SOPC) or dioleoyl phosphatidylcholine (DOPC), are used and the line tensions of pure DOPC bilayers are in the range of 3.9$27.7 \mathrm{pN}$ (summarized by Portet and Dimova, 2010). Zhelev and Needham reported that the line tension of pure SOPC bilayers is increased about 3-fold by the inclusion of cholesterol at 50 mol\% (Zhelev and Needham, 1993). Karatekin and co-workers also reported that the line tension of pure DOPC bilayers is increased about 3 -fold by the inclusion of cholesterol at $30 \mathrm{~mol} \%$ (Karatekin et al., 2003). In our MD simulations, the line tension of the pure DPPC bilayer is increased about 3- to 15-fold by the inclusion of cholesterol at $40 \mathrm{~mol} \%$ (Fig. 3B). Cholesterol effects on the line tension of the DPPC bilayer in our MD simulation are large compared to those observed for similar phospholipid bilayers investigated experimentally. We consider this difference to be acceptable because of the large variations in experimental measurements (Portet and Dimova, 2010) and the underlying effects of the types of phospholipids, which are difficult to state from the available data. This paper is, to the best of our knowledge, the first estimation of the line tension in the cholesterol-containing bilayer on the molecular scale and succeeds in determining the relationship between the cholesterol concentration and the line tension.

The line tension of the pore edge, which is an energetic loss per edge length, partially arises from the bending energy of the phospholipid monolayer of the bilayer around the pore edge (Wohlert et al., 2006). Although estimation of the bending modulus at the nanometer edge is a challenging problem (Nakamura and Shinoda, 2013), we suspect that the increase of the cholesterol concentration around the pore edge increases the bending modulus of the pore edge and the line tension (Genova et al., 2014). According to previous experimental (Mills and Toombes, 2008) and simulation studies (Róg et al., 2009), cholesterol molecules rectify the disordered orientations of hydrophobic tails of co-existing phospholipid molecules, which is one of the causes for the high bending moduli of cholesterol-containing bilayers. In our MD simulations, the cholesterol molecules are distributed around the pore edge (Fig. 4) and the cholesterol concentration around the pore edge increases with the increase of the overall cholesterol concentration (Fig. 3C). We propose that the increase of the cholesterol concentration around the pore edge increases the bending modulus of the pore edge and the line tension.

\subsection{Line tension and physical methods for drug delivery}

It is known that the cholesterol concentration varies depending strongly on the type of cell (Alberts et al., 1994). Typical cell membranes of mammals have a cholesterol concentration of 15-30 mol\% and that of the red blood cell membrane is $40-50 \mathrm{~mol} \%$. However, cholesterol is non-existent in coli bacillus membranes. With the increase of the cholesterol concentration, the line tension can increase about 3-15 fold in the MD simulation (Fig. 3B). From Lister's model for pore dynamics (Litster, 1975), an increase of the line tension means an increase of the critical pore radius $R_{C}$ $\left(=\gamma_{L} / \gamma_{S}\right)$. It is known that the tension of the bilayer also depends on the cholesterol concentration. For example, it is reported that the tension of a phospholipid bilayer containing cholesterol at $40 \mathrm{~mol} \%$ is about 2-3 times larger than that of a pure bilayer (Petelska et al., 2006). In this case, bilayers containing cholesterol at 40 mol\% can contain 1-7.5 times larger pores. Additionally, the pore closure process with the higher line tension is faster owing to the higher energetic loss to maintain the edge (Karatekin et al., 2003). In the physical methods of molecular transport through the pore, for example, sonoporation, these significant differences of the line tension and the critical pore radius $R_{C}$ might limit the size and the amount of transporting molecules without rupture of the bilayer. In fact, the transduction efficiencies by sonoporation treatment are different depending on the cell lines (Pichardo et al., 2013). Although the transduction efficiency is sensitively affected by a huge number of potential factors (Kinoshita and Hynynen, 2007), the line tension, which depends strongly on the membrane composition, especially cholesterol concentration, might be the underlying factor and will be useful for explaining the variation of the transduction efficiencies in sonoporation. However, we should note that real biological membranes are consisted of various lipids, mostly of unsaturated lipids. The bending rigidity of mono-unsaturated lipid bilayer, e.g. SOPC (Genova et al., 2014), increases with the cholesterol concentration, as of saturated phospholipid bilayer, e.g. dimyristoyl phosphatidylcholine (Duwe and Sackmann, 1990). 
It might be interesting to investigate the cholesterol effects on the line tension in unsaturated lipid bilayers, or in multicomponent lipid bilayers.

\section{Summary}

To understand the cholesterol effects on the line tension of the pore edge and the molecular details of the pore edge structure, we performed MD simulations of DPPC/Chol bilayers with a pore. The pores in the cholesterol-containing bilayers are lined with not only the DPPC molecules but also the cholesterol molecules. The cholesterol concentration in the pore edge region is almost the same as the cholesterol concentration in the whole system. Based on the free energy model of the bilayer with a pore, we estimated that the line tension of the pore edge considerably increases with the increase of the cholesterol concentration. The increasing tendency is in agreement with that observed from experiments. The increase of the line tension might arise from the increase of the bending rigidity of the bilayer around the pore edge, which is induced by the cholesterol molecules distributed around the pore edge. Additionally, we propose that the considerable difference of the line tension, depending on the bilayer compositions, might be useful to explain the large variations of the transduction efficiency observed with sonoporation treatment.

\section{Acknowledgement}

This work was supported in part by Japan Society for the Promotion of Science (JSPS) Grants-in-Aid for Scientific Research (24240073, 23686031, and 15K01284) and by Grants-in-Aid for JSPS Fellows (15J05070).

\section{References}

Abiror, I. G., Arakelyan, V. B., Chernomordik, L. V., Chizmadzhev, Y. A., Pastushenko, V. F., and Tarasevich, M. R., Electric breakdown of bilayer lipid membranes i. the main experimental facts and their qualitative discussion, Bioelectrochemistry and Bioenergetics, Vol. 6, (1979), pp.37-52.

Akimov, S. a., Mukovozov, a. a., Voronina, G. F., Chizmadzhev, Y. a., and Batishchev, O. V., Line tension and structure of through pore edge in lipid bilayer, Biochemistry (Moscow) Supplement Series A: Membrane and Cell Biology, Vol. 8, (2014), pp.297-303.

Alberts, B., Bray, D., Lewis, J., Raff, M., Roberts, K., and Watson, J. D.,Molecular biology of the cell (Third edit.). New York: Garland Publishing.

Almeida, P. F., Vaz, W. L., and Thompson, T. E., Lateral diffusion in the liquid phases of dimyristoylphosphatidylcholine/cholesterol lipid bilayers: a free volume analysis, Biochemistry, Vol. 31, (1992), pp.6739-6747.

Berendsen, H. J. C., Postma, J. P. M., Gunsteren, W. F. van, and Hermans, J., Interaction models for water in relation to protein hydration,In B. Pullman (Ed.), Intermolecular Forces (p. pp. 331). Dordrecht, The Netherlands: Reidel.

Berendsen, H. J. C., Postma, J. P. M., van Gunsteren, W. F., DiNola, A., and Haak, J. R., Molecular dynamics with coupling to an external bath, The Journal of Chemical Physics, Vol. 81, (1984), pp.3684-3690.

Berendsen, H. J. C., van del Spoel, D., and van Drunen, R., Gromacs: a message-passing parallel molecular dynamics implementation, Computer Physics Communications, Vol. 91, (1995), pp.43-56.

Berger, O., Edholm, O., and Jähnig, F., Molecular dynamics simulations of a fluid bilayer of dipalmitoylphosphatidylcholine at full hydration, constant pressure, and constant temperature, Biophysical Journal, Vol. 72, (1997), pp.2002-2013.

Bussi, G., Donadio, D., and Parrinello, M., Canonical sampling through velocity rescaling, The Journal of Chemical Physics, Vol. 126, (2007), pp.014101.

Chiu, S. W., Jakobsson, E., Mashl, R. J., and Scott, H. L., Cholesterol-induced modifications in lipid bilayers: a simulation study, Biophysical Journal, Vol. 83, (2002), pp.1842-1853.

De Joannis, J., Jiang, F. Y., and Kindt, J. T., Coarse-grained model simulations of mixed-lipid systems: composition and line tension of a stabilized bilayer edge, Langmuir: the ACS journal of surfaces and colloids, Vol. 22, (2006), pp.998-1005. 
Demel, R., The effect of sterol structure on the permeability of lipomes to glucose, glycerol and rb+, Biochimica et Biophysica Acta, Vol. 255, (1972), pp.321-330.

Duwe, H., and Sackmann, E., Bending elasticity and thermal excitations of lipid bilayer vesicles: modulation by solutes, Physica A, Vol. 163, (1990), pp.410-428.

Endress, E., Bayerl, S., Prechtel, K., Maier, C., Merkel, R., and Bayerl, T. M., The effect of cholesterol, lanosterol, and ergosterol on lecithin bilayer mechanical properties at molecular and microscopic dimensions a solid-state nmr and micropipet study, Langmuir, Vol. 18, (2002), pp.3293-3299.

Gehl, J., Electroporation: theory and methods, perspectives for drug delivery, gene therapy and research, Acta physiologica Scandinavica, Vol. 177, (2003), pp.437-447.

Genova, J., Bivas, I., and Marinov, R., Cholesterol influence on the bending elasticity of lipid membranes, Colloids and Surfaces A: Physicochemical and Engineering Aspects, Vol. 460, (2014), pp.79-82.

Gurtovenko, A. a, and Vattulainen, I., Molecular mechanism for lipid flip-flops, The journal of physical chemistry. B, Vol. 111, (2007), pp.13554-9.

Hess, B., and Kutzner, C., Gromacs 4: algorithms for highly efficient, load-balanced, and scalable molecular simulation, Journal of Chemical Theory and Computation, Vol. 4, (2008), pp.435-447.

Höltje, M., Förster, T., Brandt, B., Engels, T., von Rybinski, W., and Höltje, H. D., Molecular dynamics simulations of stratum corneum lipid models: fatty acids and cholesterol, Biochimica et Biophysica Acta, Vol. 1511, (2001), pp.156-167.

Humphrey, W., Dalke, A., and Schulten, K., Vmd: visual molecular dynamics, Journal of Molecular Graphics, Vol. 14, (1996), pp.33-38.

Karal, M. A. S., Alam, J. M., Takahashi, T., Levadny, V., and Yamazaki, M., Stretch-activated pore of the antimicrobial peptide, magainin 2, Langmuir, Vol. 31, (2015), pp.3391-3401.

Karatekin, E., Sandre, O., Guitouni, H., Borghi, N., Puech, P.-H., and Brochard-Wyart, F., Cascades of transient pores in giant vesicles: line tension and transport, Biophysical Journal, Vol. 84, (2003), pp.1734-1749.

Kinoshita, M., and Hynynen, K., Key factors that affect sonoporation efficiency in in vitro settings: the importance of standing wave in sonoporation, Biochemical and Biophysical Research Communications, Vol. 359, (2007), pp.860-865.

Kodama, T., Tomita, Y., Watanabe, Y., Koshiyama, K., Yano, T., and Fujikawa, S., Cavitation bubbles mediated molecular delivery during sonoporation, Journal of Biomechanical Science and Engineering, Vol. 4, (2009), pp.124-140.

Koshiyama, K., and Wada, S., Molecular dynamics simulations of pore formation dynamics during the rupture process of a phospholipid bilayer caused by high-speed equibiaxial stretching, Journal of Biomechanics, Vol. 44, (2011), pp.2053-2058.

Kudo, N., Okada, K., and Yamamoto, K., Sonoporation by single-shot pulsed ultrasound with microbubbles adjacent to cells., Biophysical Journal, Vol. 96, (2009), pp.4866-4876.

Leontiadou, H., Mark, A. E., and Marrink, S. J., Molecular dynamics simulations of hydrophilic pores in lipid bilayers, Biophysical journal, Vol. 86, (2004), pp.2156-2164.

Leontiadou, H., Mark, A. E., and Marrink, S.-J., Ion transport across transmembrane pores, Biophysical Journal, Vol. 92, (2007), pp.4209-4215.

Litster, J. D., Stability of lipid bilayers and red blood cell membranes, Physics Letters A, Vol. 53, (1975), pp.193-194.

Marrink, S. J., de Vries, A. H., and Tieleman, D. P., Lipids on the move: simulations of membrane pores, domains, stalks and curves, Biochimica et Biophysica Acta, Vol. 1788, (2009), pp.149-168.

Mills, T., and Toombes, G., Order parameters and areas in fluid-phase oriented lipid membranes using wide angle x-ray scattering, Biophysical Journal, Vol. 95, (2008), pp.669-681.

Nakamura, T., and Shinoda, W., Method of evaluating curvature-dependent elastic parameters for small unilamellar vesicles using molecular dynamics trajectory, Journal of Chemical Physics, Vol. 138, (2013), doi:10.1063/1.4795579

Needham, D., and Nunn, R. S., Elastic deformation and failure of lipid bilayer membranes containing cholesterol, Biophysical Journal, Vol. 58, (1990), pp.997-1009.

Petelska, A. D., Naumowicz, M., and Figaszewski, Z. a, The interfacial tension of the lipid membrane formed from lipid-cholesterol and lipid-lipid systems., Cell biochemistry and biophysics, Vol. 44, (2006), pp.205-211. 
Pichardo, S., Togtema, M., Jackson, R., Zehbe, I., and Curiel, L., Influence of cell line and cell cycle phase on sonoporation transfection efficiency in cervical carcinoma cells under the same physical conditions, IEEE Transactions on Ultrasonics, Ferroelectrics, and Frequency Control, Vol. 60, (2013), pp.432-435.

Pike, L. J., The challenge of lipid rafts, Journal of Lipid Research, Vol. April Supp, (2009), pp.S323-S328.

Portet, T., and Dimova, R., A new method for measuring edge tensions and stability of lipid bilayers: effect of membrane composition., Biophysical Journal, Vol. 99, (2010), pp.3264-3273.

Róg, T., Pasenkiewicz-Gierula, M., Vattulainen, I., and Karttunen, M., Ordering effects of cholesterol and its analogues, Biochimica et Biophysica Acta, Vol. 1788, (2009), pp.97-121.

Saito, H., and Shinoda, W., Cholesterol effect on water permeability through dppc and psm lipid bilayers: a molecular dynamics study, The Journal of Physical Chemistry. B, Vol. 115, (2011), pp.15241-15250.

Shigematsu, T., Koshiyama, K., and Wada, S., Molecular dynamics simulations of pore formation in stretched phospholipid/cholesterol bilayers, Chemistry and physics of lipids, Vol. 183, (2014), pp.43-49.

Tolpekina, T. V, den Otter, W. K., and Briels, W. J., Simulations of stable pores in membranes: system size dependence and line tension, The Journal of Chemical Physics, Vol. 121, (2004), pp.8014-8020.

Wang, H., de Joannis, J., Jiang, Y., Gaulding, J. C., Albrecht, B., Yin, F., Khanna, K., and Kindt, J. T., Bilayer edge and curvature effects on partitioning of lipids by tail length: atomistic simulations, Biophysical Journal, Vol. 95 , (2008), pp.2647-2657.

West, A., Ma, K., Chung, J. L., and Kindt, J. T., Simulation studies of structure and edge tension of lipid bilayer edges: effects of tail structure and force-field, The Journal of Physical Chemistry A, (2013). doi:10.1021/jp400371k

Wohlert, J., den Otter, W. K., Edholm, O., and Briels, W. J., Free energy of a trans-membrane pore calculated from atomistic molecular dynamics simulations, The Journal of Chemical Physics, Vol. 124, (2006), pp.154905.

Zhelev, D. V, and Needham, D., Tension-stabilized pores in giant vesicles: determination of pore size and pore line tension, Biochimica et Biophysica Acta, Vol. 1147, (1993), pp.89-104. 